\title{
A GAME-THEORETICAL MODEL OF COMPETITION FOR STAFF BETWEEN TWO DEPARTMENTS
}

\author{
A. Y. GARNAEV \\ Department of Computer Modelling and Multiprocessor Systems \\ Faculty of Applied Mathematics and Control Processes, St. Petersburg State University \\ Universitetskii prospekt 35, Peterhof, St. Petersburg 198504, Russia \\ E-mail: agarnaev@rambler.ru
}

\begin{abstract}
The main purpose of this paper is to analyze a development of a scenario suggested by Baston and Garnaev (2005) for modelling the situation where two departments in a large organization are each seeking to make an appointment within the same area of expertise, for instance, a computer science specialist. The departments are interested in three skills of the candidate (say, writing code, communication and in algorithms). In our scenario Department 1 wants to employ a candidate with excellent skills in writing code and communication. Department 2 is looking for a candidate with excellent skills in developing algorithms and communication. So, the departments have a joint interest, as well as their own interest. The scenario is modelled by a multistage non-zero sum game. Nash equilibrium and Stackelberg strategies are derived.
\end{abstract}

1. Introduction. The main purpose of this paper is to analyze a development of a scenario suggested by Baston and Garnaev (2005) for modelling the situation where two departments in a large organization are each seeking to make an appointment within the same area of expertise, for instance, a computer science specialist. The departments are interested in three skills of the candidate (say, writing code, communication and in algorithms). In our scenario Department 1 wants to employ a candidate with excellent skills in writing code and communication. Department 2 is looking for a candidate with excellent skills in developing algorithms and communication. So, the departments have a joint interest as well as their own interest. In Baston and Garnaev (2005) departments were interested only in one candidate skill. The scenario for the case of two skills in the secretary game was considered by Sakaguchi and Mazalov (2004), who investigated

2000 Mathematics Subject Classification: Primary 60C05.

Key words and phrases: secretary problem, Nash equilibrium, Stackelberg strategies, multistage non-zero sum game.

Research supported in part by Grant of Scientific School 2174.2003.1.

The paper is in final form and no version of it will be published elsewhere. 
the game in which two players (President and Vice-president) both want to employ one secretary from a set of $n$ applicants. Excellent management ability (a foreign language) is required by the President (Vice-president).

It is interesting to note that Ramsey and Szajowski (2006) applied the Baston and Garnaev game as an illustrative example in their extension of the concept of correlated strategies to Markov stopping games where the situation of multiple solutions may arise.

The main topic of this paper is strictly related to a game version of the best choice problem (BCP) (see Ferguson (1989), Freeman (1983), Kwan and Yuan Y (1988) about BCP and Sakaguchi $(1989,1995)$ about its game theoretic versions). Enns and Ferenstein (1985), Enns, Ferenstein and Sheahan (1986) have solved a non-zero sum game related to BCP. The full information version of the game has been solved by Chen, Rosenberg and Shepp (1997). The relation between the players is as follows: denote the decision maker as Player 1 and Player 2; when an item appears, then Player 1 always has the first opportunity to decide to hire the applicant or not (unless she has hired one already); one can say that Player 1 has priority; if Player 1 does not hire the current applicant, then Player 2 can decide whether to hire the applicant or not (unless she has hired one already); if neither Player hires the current applicant they interview the next applicant; the interview process continues until both players have hired an applicant; a hired applicant does not hesitate, but accepts an offer without delay and additional conditions.

Ramsey and Szajowski (2001) have considered a mathematical model of competitive stopping of a Markov chain. Two decision makers observe goods or applicants for a post in sequence. Each object is characterized by some random variable. The aim of the players is to obtain the most profitable object. The rules of the game are as follows: an object can be accepted only at the moment of its appearance; at each moment $N$ one object is presented; when both players want to accept the same object then some rules for assigning priority of acceptance to one of the players is applied; any accepted object is available with some given probability; if such an object is not available it is lost to both players forever. The solution of such games is found by solving a system of nested Bellman equations.

2. Preliminary notions for the three quality game. By choosing the units appropriately we can suppose that each applicant has three kinds of expertise (code, algorithms, communication) drawn independently at random from the interval $[0,1]$ and that the cost of a shortfall in expertise of a department not employing an applicant is $c$. We can think of the situation as a multi-stage game with the $r$-th stage being one in which rejection of the current applicant would leave $r-1$ applicants available for interview. However, following Baston and Garnaev (2005), we will adopt a naive approach and not use the general theory of such games.

Consider first the decision problem in which a player has an interest in two kinds of skills and he knows that there are $r$ applicants remaining and the other player has filled the post in his department. We will denote the expectation of such a player in this case by $u_{r}$. Clearly, if $r=1$, the player will accept the applicant and $u_{r}=1 / 4$. Furthermore, when there are $r-1$ applicants available if the current one is rejected, he will accept the 
applicant he is interviewing if the applicant has expertise $x$ and $y$ where $x y \geq u_{r-1}$ and reject otherwise. Thus

$$
u_{r}=\int_{x y>u_{r-1}} x y d x d y+\int_{x y \leq u_{r-1}} u_{r-1} d x d y=\frac{3}{4} u_{r-1}^{2}-\frac{1}{2} \ln \left(u_{r-1}\right) u_{r-1}^{2}+\frac{1}{4} .
$$

3. The three quality game with one and two applicants. We now investigate the scenario described in Section 1 where there are two departments (two players). The payoff of an individual is the product of the two skill measures the player is interested in (e.g. $x$-skills in code writing; $y$-communication skills; $z$-skills in developing algorithms. These measures are assumed to be independent. Also we assume that if both players wish to accept a candidate, the candidate accepts Player 1's offer with probability $1 / 2$, otherwise he accepts Player 2's offer.

The case when there is only one applicant presents no difficulties because both players will want to appoint as they will not want a shortfall of expertise in their department. Thus there is a unique Nash equilibrium and the expected payoff for each player is $(1 / 4-c) / 2=(1-4 c) / 8$.

The position is very different when there are two applicants. The game is now a two-stage rather than a single shot game and what is meant by a Nash equilibrium has to be considered. We will take a naive view and assume the players will play a Nash equilibrium when interviewing an individual applicant. In essence, we are restricting attention to subgame perfect Nash equilibria. When the first applicant is interviewed, each player knows that he can expect $1 / 4$ if he rejects and the other player accepts and $(1-4 c) / 8$ if both reject. We can represent the situation at the first stage by a bimatrix game $M_{2}(x, y, z)$ given by

$$
M_{2}(x, y, z)=\begin{array}{cc}
\text { Accept } & \text { Reject } \\
\text { Reject }
\end{array}\left(\begin{array}{cc}
((x y+1 / 4) / 2,(y z+1 / 4) / 2) & (x y, 1 / 4) \\
(1 / 4, y z) & ((1-4 c) / 8,(1-4 c) / 8)
\end{array}\right)
$$

Note that

(a) for $x y \geq 1 / 4$ and $y z \geq 1 / 4$ Accept dominates Reject for both players,

(b) for $x y \leq \max \{0,(1-4 c) / 8\}$ and $y z \leq \max \{0,(1-4 c) / 8\}$ Reject dominates Accept for both players,

(c) for $x y>\max \{0,(1-4 c) / 8\}$ and $y z<\max \{0,(1-4 c) / 8\}$ or $x y>1 / 4$ and $y z \in$ $(\max \{0,(1-4 c) / 8\}, 1 / 4)$ (Accept, Reject) is the unique Nash equilibrium,

(d) for $y z>\max \{0,(1-4 c) / 8\}$ and $x y<\max \{0,(1-4 c) / 8\}$ or $y z>1 / 4$ and $x y \in$ $(\max \{0,(1-4 c) / 8\}, 1 / 4)$ (Reject, Accept) is the unique Nash equilibrium,

(e) for $x y, y z \in[\max \{0,(1-4 c) / 8\}, 1 / 4]$ there are two pure Nash equilibria, namely (Accept, Reject) and (Reject, Accept) and one mixed in which Player 1 accepts an applicant with probability $(4 c-1+8 y z) /(4(y z+c))$ and Player 2 accepts an applicant with probability $(4 c-1+8 x y) /(4(x y+c))$. The payoff vector for the mixed strategy pair is given by $\left(v_{1}, w_{1}\right)$ where

$$
v_{1}=\frac{(12+16 c) x y+4 c-1}{32(x y+c)}, \quad w_{1}=\frac{(12+16 c) y z+4 c-1}{32(y z+c)} .
$$


Of course neither of the pure Nash equilibria are symmetric and this could be seen as a drawback, because the scenario does not present any differences between the players. However, we should not dismiss these equilibria out of hand as they could arise from different dynamics in the organization. For instance, if one departmental head is somewhat aggressive and the other more easy-going, it is quite likely that the more aggressive head would state what he was going to do; in essence the aggressive head assumes the role of a Stackelberg leader. The mixed Nash equilibrium has the advantage that each player adopts the same strategy so that it can be argued that it is fairer than either of the pure equilibria, but it has the drawback that the players use complicated probabilities to decide their actions.

So far we have looked at the situation where the players are actually confronted with the applicant's expertise. However, from a game theoretic point of view, the strategies should be announced before the interview takes place; in other words, we should define a function $s:[0,1] \times[0,1] \rightarrow[0,1]$ for each player where $s(x, y)$ represents the probability of accepting an applicant with two kinds of expertise of interest $x$ and $y$. Of course it is reasonable to assume that if a player accepts an applicant with expertise $x$ and $y$, then it would appear unreasonable to expect the player to reject an applicant of expertise $x^{\prime}$ and $y^{\prime}$ where $x^{\prime} y^{\prime}<x y$. If this condition is imposed, there are just two pure Nash equilibria for the players; one Player accepts the first applicant if and only if the applicant has two kinds of expertise of interest $x$ and $y$ such that $x y \geq 1 / 4$ and the other Player accepts if and only if the applicant has two kinds of expertise of interest $y$ and $z$ such that $y z \geq \max \{(1-4 c) / 8,0\}$. Note that, at these Nash equilibria, both applicants will be accepted if $c \geq 1 / 4$ but that, for $c<1 / 4$, there is a positive probability that only one applicant will be accepted.

We now calculate the expectated values for the strategies at which Player 1 (the Stackelberg leader) accepts an applicant if and only if the product of two kinds of expertise in which he has interest is at least $1 / 4$ and Player 2 (the Stackelberg follower) accepts if and only if the corresponding product is at least $(1-4 c) / 8$. We consider two cases: (a) $c \leq 1 / 4$ and $(\mathrm{b}) c>1 / 4$.

(a) Assuming $c \leq 1 / 4$, we have that the payoffs $V_{2}(c)$ and $W_{2}(c)$ of Player 1 and Player 2 are given as follows

$$
\begin{aligned}
V_{2}= & \int_{1 / 4}^{1} \int_{(1-4 c) /(8 y)}^{1} \int_{1 /(4 y)}^{1} \frac{4 x y+1}{8} d x d z d y \\
& +\int_{1 / 4}^{1} \int_{0}^{(1-4 c) /(8 y)} \int_{1 /(4 y)}^{1} x y d x d z d y \\
& +\int_{(1-4 c) / 8}^{1 / 4} \int_{(1-4 c) /(8 y)}^{1} \int_{0}^{1} \frac{1}{4} d x d z d y+\int_{1 / 4}^{1} \int_{(1-4 c) /(8 y)}^{1} \int_{0}^{1 /(4 y)} \frac{1}{4} d x d z d y \\
& +\int_{0}^{(1-4 c) / 8} \int_{0}^{1} \int_{0}^{1} \frac{1-4 c}{8} d x d z d y+\int_{(1-4 c) / 8}^{1-4 c) / 8 y} \int_{0}^{1} \frac{1-4 c}{8} d x d z d y \\
& +\int_{1 / 4}^{1} \int_{0}^{(1-4 c) / 8 y} \int_{0}^{1 /(4 y)} \frac{1-4 c}{8} d x d z d y
\end{aligned}
$$




$$
\begin{aligned}
= & \frac{141}{512}-\frac{\ln (2)}{64}+\left(\frac{\ln (2)}{8}-\frac{15}{128}\right) c+\left(\frac{7}{16}+\frac{\ln (2)}{4}\right) c^{2} \\
& +\frac{1}{64} \ln (1-4 c)-\frac{1}{4} \ln (1-4 c) c^{2}, \\
W_{2}= & \int_{1 / 4}^{1} \int_{(1-4 c) /(8 y)}^{1} \int_{1 /(4 y)}^{1} \frac{4 y z+1}{8} d x d z d y \\
& +\int_{1 / 4}^{1} \int_{0}^{(1-4 c) /(8 y)} \int_{1 /(4 y)}^{1} \frac{1}{4} d x d z d y \\
& +\int_{(1-4 c) / 8}^{1 / 4} \int_{(1-4 c) /(8 y)}^{1} \int_{0}^{1} y z d x d z d y+\int_{1 / 4}^{1} \int_{(1-4 c) /(8 y)}^{1} \int_{0}^{1 /(4 y)} y z d x d z d y \\
& +\int_{0}^{(1-4 c) / 8} \int_{0}^{1} \frac{1-4 c}{8} d x d z d y+\int_{(1-4 c) / 8}^{1 / 4} \int_{0}^{1} \frac{1-4 c}{8} d x d z d y \\
& +\int_{1 / 4}^{1} \int_{0}^{(1-4 c) / 8 y} \int_{0}^{1 /(4 y)} \frac{1-4 c}{8} d x d z d y \\
= & \frac{289}{1024}-\frac{\ln (2)}{32}-\left(\frac{15}{128}+\frac{\ln (2)}{8}\right) c-\frac{1}{128} \ln (1-4 c)+\frac{1}{16} c \ln (1-4 c) \\
& +\frac{21}{64} c^{2}-\frac{1}{8} c^{2} \ln (1-4 c) .
\end{aligned}
$$

It is quite surprising that the function $V_{2}(c)$ is concave on $[0,1 / 4]$ : it is decreasing on $\left[0, c_{*}\right]$ and it is increasing on $\left[c_{*}, 1 / 4\right]$ where $c_{*}=0.079794$. Thus, $c_{*}$ is the most unfavorable shortfall payoff for the Leading player, meanwhile $1 / 4$ is his most favorable shortfall payoff. The Follower player payoff $W_{2}$ is decreasing on $[0,1 / 4]$. Thus, the most favorable shortfall payoff for the Leader player is also the most Follower player unfavorable one. Also,

$$
\begin{aligned}
V_{2}(0) & =\frac{141}{512}-\frac{\ln (2)}{64}=0.26456, \\
\lim _{c \rightarrow 1 / 4} V_{2}(c) & =\frac{35}{128}+\frac{\ln (2)}{32}=0.295098, \\
V_{2}\left(c_{v *}\right) & =0.260617 \\
W_{2}(0) & =\frac{289}{1024}-\frac{3 \ln (2)}{128}=0.26056, \\
\lim _{c \rightarrow 1 / 4} W_{2}(c) & =\frac{35}{128}-\frac{\ln (2)}{16}=0.2305116 .
\end{aligned}
$$

To find how much the Stackelberg leader can gain from his leading strategy we have to find $V_{2}(c)-W_{2}(c)$ which is given as follows

$$
\begin{aligned}
V_{2}(c)-W_{2}(c)= & \frac{\ln (2)}{64}-\frac{7}{1024}+\frac{1}{4} \ln (2) c+\left(\frac{7}{64}+\frac{\ln (2)}{4}\right) c^{2}+\frac{3}{128} \ln (1-4 c) \\
& -\frac{1}{16} c \ln (1-4 c)-\frac{1}{8} c^{2} \ln (1-4 c) .
\end{aligned}
$$

It is clear that $V_{2}(c)-W_{2}(c)>0$ for $c \in[0,1 / 4]$ and $V_{2}(c)-W_{2}(c)$ is an increasing function on $[0,1 / 4]$ which increases from $-7 / 1024+\ln (2) / 64=0.003994$ for $c=0$ to 
$3 \ln (2) / 32=0.064983$ for $c=1 / 4$. Thus, at $c=1 / 4$ the Stackelberg leader gains 16.27 times more that at $c=0$.

The joint payoff of Players are given as follows

$$
\begin{aligned}
V_{2}(c)+W_{2}(c)= & \frac{1}{128} \ln (1-4 c)+\frac{1}{16} c \ln (1-4 c)-\frac{3}{8} c^{2} \ln (1-4 c) \\
& +\left(\frac{49}{64}+\frac{\ln (2)}{4}\right) c^{2}-\frac{15}{64} c-\frac{3}{64} \ln (2)+\frac{571}{1024}
\end{aligned}
$$

It is clear that $V_{2}(c)+W_{2}(c)$ is a concave function which gets its minimum at $c_{* *}=$ 0.147861 and

$$
\begin{aligned}
V_{2}\left(c_{* *}\right)+W_{2}\left(c_{* *}\right) & =0.503572, \\
V_{2}(0)+W_{2}(0) & =\frac{571}{1024}-\frac{3 \ln (2)}{64}=0.525126, \\
\lim _{c \rightarrow 1 / 4} V_{2}(c)+W_{2}(c) & =\frac{35}{64}-\frac{\ln (2)}{32}=0.525214 .
\end{aligned}
$$

(b) Assuming $c>1 / 4$, we have that the payoffs $V_{2}(c)$ and $W_{2}(c)$ of player 1 and player 2 are independent of $c$ and they are given as follows

$$
\begin{aligned}
V_{2}(c) & =\lim _{c \rightarrow 1 / 4} V_{2}(c)=\frac{35}{128}+\frac{\ln (2)}{32}=0.295098, \\
W_{2}(c) & =\lim _{c \rightarrow 1 / 4} W_{2}(c)=\frac{35}{128}-\frac{\ln (2)}{16}=0.2305116 .
\end{aligned}
$$

4. The general case of three quality game. We saw in the last section that the two applicant game presented difficulties so we must expect similar ones to occur in the general game. However, when there are three or more applicants, further complications arise as it was pointed out by Baston and Garnaev (2005) for the One Quality Game. In the two applicant game, the players know their expectations if both players reject the first applicant but, when there are three applicants and both reject the first applicant, their expectations will depend on which Nash equilibrium is adopted at the second stage. We will assume that there is some kind of consistency in the actions of the players at the various stages and investigate a number of scenarios to see how the payoffs differ and how they are affected by the value of $c$. In the two applicant case, we have suggested that, it might benefit the players to agree to randomize who takes the role of Stackelberg leader so we will consider the situation in which, at each stage, the players agree to randomize who takes that role. By doing this the players create a symmetric situation where, at each stage, the players have the same expectation if both players reject the current applicant. It is therefore very different from the situation in which the players agree to take it in turns to assume the role of Stackelberg leader; in this case, at any given stage, the players will in general have different expectations. If there is no agreement to randomize concerning who takes the role of Stacklberg leader, it seems natural to assume that a player who adopts the role of a Stackelberg leader for one stage would take that role at every stage; we investigate how unfair this is on the Stackelberg follower. 
Assuming Players 1 and 2 believe their expectations are respectively $V_{r-1}$ and $W_{r-1}$ if they both reject the applicant interviewed at stage $r$, the $r$-stage bimatrix is

Accept

$$
M_{r}(x)=\begin{array}{lc}
\operatorname{Accept} \\
\operatorname{Reject}
\end{array}\left(\begin{array}{cc}
\left(\left(x y+u_{r-1}\right) / 2,\left(y z+u_{r-1}\right) / 2\right) & \left(x y, u_{r-1}\right) \\
\left(u_{r-1}, y z\right) & \left(V_{r-1}, W_{r-1}\right)
\end{array}\right)
$$

The result of the following lemma enables us to carry out a similar analysis to that of the two applicant case.

LEMma. For all $r, u_{r} \geq \max \left\{V_{r}, W_{r}\right\}$.

Proof. For any strategy pair in a two-person non-zero sum game, the expectations in a Nash equilibrium are non-decreasing functions of the elements in the matrix. Because $u_{1}=1 / 4 \geq(1-4 c) / 8$, the result follows by induction.

We now calculate the expectations for the strategies in which Player 1 (the Stackelberg leader) accepts an applicant if and only if the product of his two kinds of expertise is at least $u_{r-1}$ and Player 2 (the Stackelberg follower) accepts if and only if this product is at least $W_{r-1}$. Then

$$
\begin{aligned}
& V_{r}=\int_{u_{r-1}}^{1} \int_{W_{r-1} / y}^{1} \int_{u_{r-1} / y}^{1} \frac{4 x y+1}{8} d x d z d y \\
& +\int_{u_{r-1}}^{1} \int_{0}^{W_{r-1} / y} \int_{u_{r-1} / y}^{1} x y d x d z d y \\
& +\int_{W_{r-1}}^{u_{r-1}} \int_{W_{r-1} / y}^{1} \int_{0}^{1} u_{r-1} d x d z d y+\int_{u_{r-1}}^{1} \int_{W_{r-1} / y}^{1} \int_{0}^{u_{r-1} / y} u_{r-1} d x d z d y \\
& +\int_{0}^{W_{r-1}} \int_{0}^{1} \int_{0}^{1} V_{r-1} d x d z d y+\int_{W_{r-1}}^{u_{r-1}} \int_{0}^{W_{r-1} / y} \int_{0}^{1} V_{r-1} d x d z d y \\
& +\int_{u_{r-1}}^{1} \int_{0}^{W_{r-1} / y} \int_{0}^{u_{r-1} / y} V_{r-1} d x d z d y= \\
& =-\frac{3}{4} u_{r-1}^{2} \ln \left(u_{r-1}\right)+\frac{1}{8} W_{r-1} \ln \left(u_{r-1}\right)-\frac{21}{8} W_{r-1} u_{r-1} \\
& +\frac{7}{8} u_{r-1}^{2}-\frac{1}{8} u_{r-1}+\frac{1}{8} u_{r-1} \ln \left(u_{r-1}\right)+\frac{3}{8} W_{r-1}+\frac{5}{4} u_{r-1}^{2} W_{r-1} \\
& +\frac{1}{4}+u_{r-1} W_{r-1} \ln \left(W_{r-1}\right)-u_{r-1} W_{r-1} \ln \left(u_{r-1}\right) \\
& +\left(2 W_{r-1}-\ln \left(W_{r-1}\right) W_{r-1}+W_{r-1} \ln \left(u_{r-1}\right)-u_{r-1} W_{r-1}\right) V_{r-1} \text {, } \\
& W_{r}=\int_{u_{r-1}}^{1} \int_{W_{r-1} / y}^{1} \int_{u_{r-1} / y}^{1} \frac{4 y z+1}{8} d x d z d y \\
& +\int_{u_{r-1}}^{1} \int_{0}^{W_{r-1} / y} \int_{u_{r-1} / y}^{1} u_{r-1} d x d z d y \\
& +\int_{W_{r-1}}^{u_{r-1}} \int_{W_{r-1} / y}^{1} \int_{0}^{1} y z d x d z d y+\int_{u_{r-1}}^{1} \int_{W_{r-1} / y}^{1} \int_{0}^{u_{r-1} / y} y z d x d z d y
\end{aligned}
$$




$$
\begin{aligned}
& +\int_{0}^{W_{r-1}} \int_{0}^{1} \int_{0}^{1} W_{r-1} d x d z d y+\int_{W_{r-1}}^{u_{r-1}} \int_{0}^{W_{r-1} / y} \int_{0}^{1} W_{r-1} d x d z d y \\
& +\int_{u_{r-1}}^{1} \int_{0}^{W_{r-1} / y} \int_{0}^{u_{r-1} / y} W_{r-1} d x d z d y= \\
& =\frac{1}{8} u_{r-1}+\frac{1}{8} \ln \left(u_{r-1}\right)-\frac{1}{8} u_{r-1}^{2}+\frac{1}{4} \\
& +\left(\frac{3}{2}-\frac{3}{4} u_{r-1}+\frac{3}{4} \ln \left(u_{r-1}\right)-\frac{1}{2} \ln \left(W_{r-1}\right)\right) W_{r-1}^{2} \\
& +\left(\frac{1}{8}-\frac{9}{8} u_{r-1}-u_{r-1} \ln \left(u_{r-1}\right)+u_{r-1}^{2}+\frac{1}{8} \ln \left(u_{r-1}\right)\right) W_{r-1} .
\end{aligned}
$$

5. Conclusion. Using the results of Section 4 we can find $V_{r}$ and $W_{r}$ for any $r$. For example for $r \in[3,6]$ if $c \geq 1 / 4$ then $V_{r}$ and $W_{r}$ are constant on $c$ and for $c \in[0,1 / 4]$ they are given as follows:

$\begin{array}{ccccccc}c & 0 & 0.05 & 0.1 & 0.15 & 0.2 & 0.25 \\ V_{2} & 0.2646 & 0.2612 & 0.2609 & 0.2646 & 0.2738 & 0.2951 \\ V_{3} & 0.3332 & 0.3317 & 0.3317 & 0.3336 & 0.3381 & 0.3482 \\ V_{4} & 0.3813 & 0.3803 & 0.3803 & 0.3814 & 0.3841 & 0.3902 \\ V_{5} & 0.4186 & 0.4179 & .4178 & 0.4186 & 0.4204 & 0.4246 \\ V_{6} & 0.4491 & .4486 & 0.4485 & 0.4490 & 0.4503 & 0.4533 \\ W_{2} & 0.2605 & 0.2523 & 0.2449 & 0.2385 & 0.2334 & 0.2301 \\ W_{3} & 0.3702 & 0.3645 & 0.3595 & 0.3552 & 0.3518 & 0.3486 \\ W_{4} & 0.4444 & .4401 & 0.4364 & 0.4331 & 0.4306 & 0.4289 \\ W_{5} & 0.4989 & 0.4955 & 0.4924 & 0.4899 & 0.4879 & 0.4866 \\ W_{6} & 0.5409 & .5381 & 0.5356 & 0.5335 & 0.5319 & 0.5308\end{array}$

In Section 3 we saw that, when there are just two applicants, then the payoff of the Stackelberg leader is a concave function on $c \in[1,1 / 4]$ while the Follower player payoff is decreasing on $[0,1 / 4]$. Also it was shown that the most favorable shortfall payoff for the Leader player is also the most Follower player unfavorable one. As it could be seen from the above table the same pattern holds for more than two applicants.

\section{References}

V. Baston and A. Garnaev (2005), Competition for staff between two departments, in: Game Theory and Applications. Vol. X, Nova Sci. Publ., Commack, NY, 27-36.

R. W. Chen, B. Rosenberg and L. A. Shepp (1997), A secretary problem with two decision makers, J. Appl. Probab. 34, 1068-1074.

Y. H. Chun (1992), On the sequential selection problem, Decision Sciences 23, 1251-1262.

Y. H. Chun (1999), Selecting the best choice in the full information group interview problem, Eur. J. Oper. Res. 119, 635-651.

Y. H. Chun (1996), Selecting the best choice in the weighted secretary problem, Eur. J. Oper. Res. 92, 135-147.

E. G. Enns and E. Ferenstein (1985), The horse game, J. Oper. Res. Soc. Jap. 28, 51-62. 
E. G. Enns, E. Z. Ferenstein and J. N. Sheahan (1986), A curious recursion arising in game theory, Utilitas Math. 30, 219-228.

T. S. Ferguson (1989), Who solved the secretary problem?, Statistical Science 4, 282-296.

P. R. Freeman (1983), The secretary problem and its extensions: a review, Int. Statist. Rev. 51, 189-206.

C. C. Y. Kwan and Y. Yuan (1988), A sequential selection problem, Decision Sciences 19, 762-770.

D. Ramsey and K. Szajowski (2001), Random assignment and uncertain employment in optimal stopping of Markov processes, Game Theory and Applications 7, 147-157.

D. Ramsey and K. Szajowski (2004), Bilateral approach to the secretary problem, Annals of ISDG 7 (to appear).

D. Ramsey and K. Szajowski (2006), Correlate equilibria in competitive staff selection problem, this volume, 253-265.

M. Sakaguchi (1989), Multiperson multilateral secretary problem, Mathematica Japonica 35, 459473.

M. Sakaguchi (1995), Optimal stopping games: a review, Math. Japonica 42, 343-351.

M. Sakaguchi and V. Mazalov (2004), A non-zero-sum no-information best-choice game, Mathematical Methods of Operations Research 60, to appear. 